\title{
Etiology, Clinical Profile, Evaluation, and Management of Stridor in Children
}

\author{
Sibabratta Patnaik $^{1}$ (1) - Gifty Zacharias ${ }^{2} \cdot$ Mukesh Kumar Jain $^{1} \cdot$ K. K. Samantaray ${ }^{2} \cdot$ Sai Poojyata Surapaneni $^{2}$
}

Received: 21 July 2020 / Accepted: 25 February 2021 / Published online: 17 March 2021

(C) Dr. K C Chaudhuri Foundation 2021

\begin{abstract}
Objective To evaluate various causes of pediatric stridor and their management among admitted patients in last $2 \mathrm{y}$. Methods Retrospective study of 67 stridor cases in pediatric age group (from birth to $18 \mathrm{y}$ ), admitted to the Department of Pediatrics and ENT (Ear, Nose and Throat) from May 2018 to April 2020 were included in the study. Data were obtained from medical records regarding age, gender, clinical presentation, and management.

Results Out of 67 cases of pediatric stridor, $28.3 \%$ were infants, $50.7 \%$ were between 1 to $5 \mathrm{y}$, while $20.9 \%$ were between 5 to 18 y. Foreign body trachea (FB) was the most common (38.8\%) cause of stridor. The commonest cause of stridor among infants was laryngomalacia (47.4\%) while FB trachea (55.9\%) was the commonest cause among 1 to 5 y age group. In age group between 5 to $18 \mathrm{y}$, peritonsillar abscess and bacterial tracheitis $(21.4 \%$ each) were found to be the most common. Primary management with securing of airways were done in all cases. Curative treatment was provided according to the underlying pathology. Eight patients (11.9\%) required tracheostomy to bypass airway obstruction. There was no mortality in the present study population.

Conclusion Pediatric stridor management is a teamwork between ENT surgeons, pediatricians, and anaesthetists. Management starts with suspicion from history followed by clinical and radiological evaluation. Securing airway is of utmost importance and precise management of cause is carried out later.
\end{abstract}

Keywords Stridor $\cdot$ Laryngomalacia $\cdot$ Fiberoptic laryngoscopy $\cdot$ Tracheostomy

\section{Introduction}

Stridor is defined as a high pitch noise that arises from compromised airway at the level of larynx and trachea. The word stridor comes from the Latin word "stridulus" which means creaking, whistling, or grating noise [1]. Stridor can be inspiratory, expiratory, or biphasic. The inspiratory stridor is seen in obstruction above glottis (extrathoracic) whereas expiratory stridor is seen in obstruction of lower trachea (intrathoracic) and biphasic stridor in glottic or supraglottic pathologies (extrathoracic or intrathoracic). According to Holinger, as the diameter of larynx is small in children, even mild circumferential edema in the infraglottic region drastically reduces

Sibabratta Patnaik

drsbpatnaik45@gmail.com

1 Department of Pediatrics, Kalinga Institute of Medical Sciences, Bhubaneswar, Odisha 751024, India

2 Department of ENT, Kalinga Institute of Medical Sciences, Bhubaneswar, Odisha, India the airway lumen, resulting in stridor [2]. All patients presenting with stridor require immediate attention and evaluation of the underlying cause. In a previous study, upper airway obstruction, which presented with stridor, was responsible for $3.3 \%$ of pediatric intensive care unit (PICU) admission [3].

The literature on pediatric stridor is limited in India; therefore, this study was conducted to know the etiology, clinical presentation, along with evaluation and management of stridor.

\section{Material and Methods}

This is a retrospective record-based study of 67 stridor cases (Table 1) admitted to the Department of ENT (Ear, Nose and Throat) and Pediatrics during May 2018 to April 2020 in Kalinga Institute of Medical Sciences, Bhubaneswar, Odisha. After the approval of institutional ethics committee, medical records of all pediatric stridor cases and recorded videos of their interventional procedures were evaluated. All patients less than $18 \mathrm{y}$ admitted to hospital with stridor were 
Table 1 Children presenting with stridor in various age groups

\begin{tabular}{lllll}
\hline Causes of stridor in children & $0 \mathrm{~d}$ to $1 \mathrm{y}$ & $1 \mathrm{y}$ to $5 \mathrm{y}$ & More than 5 y to $18 \mathrm{y}$ & Total \\
\hline FB trachea & 5 & 19 & 2 & $26(38.80 \%)$ \\
Laryngomalacia & 9 & 3 & - & $12(17.91 \%)$ \\
Laryngotracheobronchitis & 4 & 5 & 1 & $10(14.92 \%)$ \\
Angioedema & - & 1 & - & $1(1.49 \%)$ \\
Subglottic stenosis & - & 1 & 2 & $3(4.47 \%)$ \\
Bacterial tracheitis & - & - & 3 & $3(4.47 \%)$ \\
Laryngeal web & - & 1 & - & $1(1.49 \%)$ \\
B/L vocal fold palsy & 1 & - & 1 & $2(2.98 \%)$ \\
Laryngeal papilloma & - & 2 & 1 & $3(4.47 \%)$ \\
Retropharyngeal/peritonsillar abscess & - & 1 & 3 & $4(5.97 \%)$ \\
Tumor & - & - & 1 & $1(1.49 \%)$ \\
Neurogenic stridor & - & 1 & - & $1(1.49 \%)$ \\
Total & 19 & 34 & 14 & 67 \\
\hline
\end{tabular}

$B / L$ Bilateral; $F B$ Foreign body included in the study. Immediate post-extubation stridor in children was excluded. Data were entered to Excel spread sheet and analyzed. Results were expressed in percentage.

Careful history from parents was mandatory and it was taken as per Table 2. The signs that had been looked for were the child's appearance, posture, drooling, craniofacial abnormalities, neck swellings, vitals (respiratory rate, pulse rate, oxygen saturation, blood pressure, and temperature), bilateral chest movements, and chest auscultation.

As majority of the patients presented to the emergency department with acute respiratory distress, airway stabilization was given first priority. Further evaluation of underlying cause and definitive management was carried out in all patients. Oxygen by prongs, high flow nasal cannula (HFNC), continuous positive airway pressure (CPAP), endotracheal intubation, and even tracheostomy were required according to the severity of disease. Once the child was stable, radiological investigations were proceeded. Chest and neck radiography (AP and lateral view), computed tomography (CT) and magnetic resonance imaging (MRI) of neck and thorax were carried out depending upon the suspicion of underlying

Table 2 Relevant history to be asked in stridor

- Onset and progression of the disease (acute/chronic)

- Age of onset of symptoms

- Feeding history (cough, vomiting, choking or cyanosis during feeding, types of food)

- Associated symptoms (fever, cough, change in voice, feeding, and stress)

- Prenatal and obstetric history

- Any co-morbidities/congenital abnormalities

- Vaccination history (Haemophilus influenzae)

- Exposure to any allergen

- Medical records to previous investigations and treatment if available. pathology. Even though radiological investigations were negative in some cases of foreign body aspiration, diagnostic bronchoscopy was proceeded. Suspected vascular abnormalities require CT or MR angiogram to confirm the diagnosis Routine complete blood count, blood smear, inflammatory markers, and coagulation profile were carried out. Definitive treatment was instituted according to diagnosis.

\section{Results}

In this retrospective evaluation of pediatric stridor, out of 67 cases, $59.7 \%$ were male. The most common age group was children between 1 to 5 y $(50.7 \%)$. Out of all children presenting with stridor, foreign body (FB) was found in $38.8 \%$ cases, while laryngomalacia and croup was found in $17.9 \%$ and $14.9 \%$ children, respectively. The commonest cause of stridor in infants was laryngomalacia (47.4\%), whereas in 1 to $5 \mathrm{y}$ children, it was foreign body (55.9\%). In 5 to 18 y age group, peritonsillar abscess and bacterial tracheitis (21.4\% each) were found to be the most common. There were 4 cases (6\%) of peritonsillar abscess, who presented with stridor. Interval tonsillectomy was required for $2(50 \%)$ cases. Ten cases of acute laryngotracheobronchitis (LTB) were found, 8 responded to conservative management and $2(20 \%)$ required mechanical ventilation. Foreign body (FB) in the airway was found in $26(38.8 \%)$ cases. Most of the cases $(80.7 \%)$ of FB were found in above $1 \mathrm{y}$ of age, vegetative FB being the commonest. Rigid bronchoscopy under general anesthesia was done for removal of FB in $25(96 \%)$ cases, while tracheostomy was required in one case. Out of 12 cases of laryngomalacia, 9 babies presented in neonatal period. All laryngomalacia cases were managed conservatively, 2 required CPAP while another 1 required HFNC. Three cases 
of bacterial tracheitis presented as stridor, all required intubation and mechanical ventilation along with IV antibiotics. Laryngeal papilloma presenting with stridor was found in 3 cases, and the mean age of presentation was $4.6 \mathrm{y}$. Vocal cord palsy (VCP) was found in 3 children; 1 in a case of ArnoldChiari malformation, 1 in a neonate with birth trauma while 1 in a child with road traffic accident (RTA). All required emergency tracheostomy. One case of anterior mediastinal mass also presented with stridor.

Eight children (11.9\%) required tracheostomy (Table 3). Nine required intubation, 2 required nasal CPAP and 2 required HFNC. Surgical intervention was required in 32 cases (47.76\%) (foreign body trachea, laryngeal papilloma, ArnoldChiari malformation, bilateral VCP, and laryngeal web).

There was no mortality in the present study, while 1 child left against medical advice.

\section{Discussion}

Stridor is a harsh, vibratory sound usually produced during inspiration, as a result of partial obstruction of airway which causes turbulent airflow through respiratory passages. As compared to adults, support components of airway in children are less developed and more compliant. Intercostal muscles, accessory muscle, and cartilaginous and perpendicular character of thoracic ribs reduce the mechanical efficiency of chest wall. They mainly rely on diaphragm for inspiration. Therefore, children are more vulnerable for airway diseases and they deteriorate very quickly if proper medical attention is not given. Because of narrow airway, stridor may be life threatening in children and need urgent intervention. The procedures like bronchoscopy, tracheostomy, intubation, and different airway surgery are challenging in children because of narrow airway, lack of adequate surgical field, risk of anesthesia, and need of expertise. Even though there are many differential diagnoses, foreign body aspiration is the commonest cause of stridor in childhood, while laryngomalacia is the commonest cause of congenital stridor [4].

LTB was commonly seen in 6 mo to 3 y age group (6 among 10 cases). Similar results were found in previous studies [5]. Viral croup constitutes more than $80 \%$ of cases of acute stridor in children with parainfluenza virus as the

Table 3 Number of tracheostomy performed

\begin{tabular}{ll}
\hline Foreign body trachea & 1 \\
Bilateral vocal cord palsy & 2 \\
Subglottic stenosis & 3 \\
Neurogenic stridor & 1 \\
Laryngeal web & 1 \\
Total number & 8 \\
\hline
\end{tabular}

commonest causative agent. All children suspected with LTB were categorized according to croup scoring system. Oxygen, nebulized epinephrine, and steroids were given as initial medical management. Patients with no improvement were considered for intubation. In the present study, $2(20 \%)$ required ventilator support. In a retrospective study, Gelbart et al. collected data on LTB between 2005 and 2012, from Royal Children's Hospital, Melbourne; 2188 children required hospitalization, $185(8.46 \%)$ of which required intensive care unit (ICU), and 65 (only $3 \%$ ) required intubation [6].

FB aspiration presents with sudden onset stridor and may not always have a suggestive history. Out of 26 cases of FB, $73.1 \%$ were between 1 and $5 \mathrm{y}$ age group and had male predominance $(57.6 \%)$. Study by Korlacki et al. and Shivakumar et al. (in Indian population) also showed a male predominance with average age as $5.5 \mathrm{y}$ and $1-3$ y respectively $[7,8]$. The diagnosis requires a high index of suspicion as foreign body intake is usually unwitnessed and children will be of preverbal age group. Common FB of bronchus were vegetative materials (Fig. 1a and b) and in 3 cases sharp objects like nail and paper pin were found. Though flexible bronchoscopy is considered effective and safe, it requires more skill. All the cases in the present study underwent rigid bronchoscopy with jet ventilation support under general anesthesia. In an infant with peanut in trachea, tracheostomy was done for attaining surgical access as the FB was tightly impacted. Previous studies have also revealed use of emergency tracheostomy for FB removal [9]. According to Ramirez-Figueroa et al., bronchoscopy is found to be $>95 \%$ effective in removal of FB [10].

Laryngomalacia is the most common congenital anomaly causing stridor due to decreased laryngeal tone. Vijayasekaran et al. observed that symptoms usually appear by $10 \mathrm{~d}$ of life and worsen during the first few months [11]. This was consistent in the present study showing 9 out of 12 (75\%) cases in the same period. In all cases, diagnosis was confirmed with flexible laryngoscopy done at 2 mo of age. They were all, managed conservatively; 2 neonates required nasal CPAP and another required HFNC. In a study by Fattah et al., conservative treatment was effective in $47(81 \%)$ whereas 11 case (19\%) required surgical treatment (supraglottoplasty) [12]. Thus, supraglottoplasty can be considered in severe cases and different series of laryngomalacia reported its success rate from $80 \%-100 \%$ [13]. In a recent study from Army Hospital Research and Referral, New Delhi; from a series of 15 cases of stridor, 4 cases were of laryngomalacia and all 4 underwent supraglottoplasty [14]. However, it is from a surgical unit, so only cases requiring surgery might be admitted. In the present series, none had evidence of bacterial infection and they required respiratory support for 3-4 d. The authors did not have the follow-up of these patients. In the authors' center supraglottoplasty has not been done till now.

There were 4 cases of peritonsillar abscess, who had stridor along with symptoms of sore throat, difficulty in swallowing, 

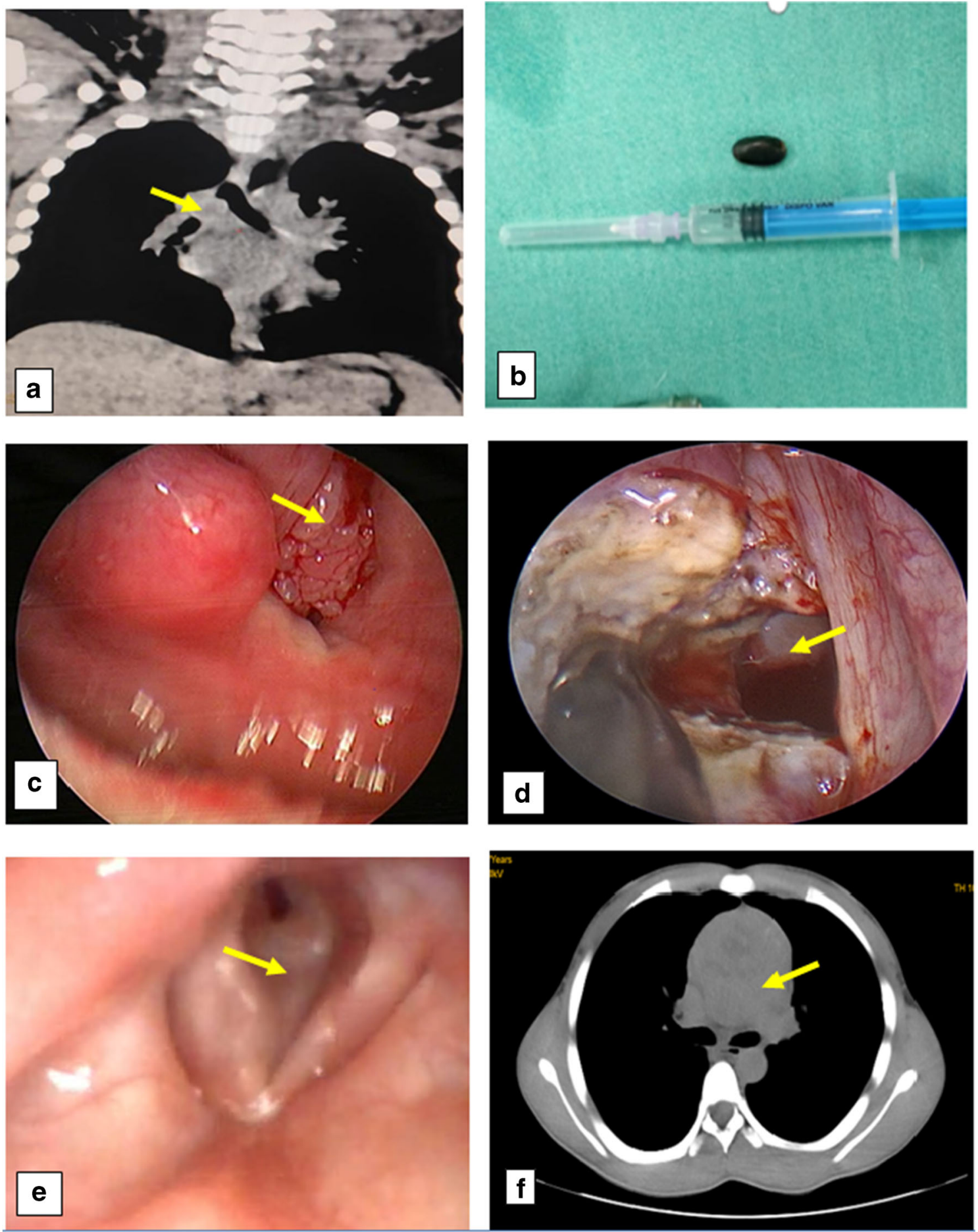

Fig. 1 a, b FB of right bronchus and the seed taken out using rigid bronchoscopy. c Papilloma of right vocal fold obstructing the entire laryngeal inlet. d Adequate airway after Kashima operation in $\mathrm{B} / \mathrm{L}$ vocal

trismus, and lymphadenopathy. Though the study by Watkinson et al. [15] shows group A beta-hemolytic streptococcus and anaerobic bacteria like Fusobacterium necrophorum and Streptococcus milleri as the commonest causative organisms, no organisms could be detected in the present study. All were cord palsy. e Grade 3 subglottic stenosis. f Upper mediastinal mass compressing the airway. $B / L$ Bilateral; $F B$ Foreign body

treated with incision and drainage and intravenous antibiotics. Two $(50 \%)$ underwent interval tonsillectomy after $6 \mathrm{wk}$, because of recurrence. Interval tonsillectomy after $6 \mathrm{wk}$ is the standard treatment for peritonsillar abscess according to Naik et al. with better postoperative pain score [16]. 
Laryngeal papilloma is the most common benign neoplasm of larynx in children with high recurrence rate. There were 3 cases with mean age of $4.7 \mathrm{y}$, who presented with complaints of inspiratory stridor, hoarseness, and progressive respiratory distress. In the study by James et al. the median age of onset was 6 y [17]. Flexible laryngoscopy showed multiple grapeslike lesions in vocal cord (Fig. 1c). Endolaryngeal surgery was performed where papilloma was dissected with coblation and diagnosis was confirmed with histopathological examination. According to Cole et al. tracheostomy is undesirable to avoid dissemination of papilloma but can be done in patients with upper respiratory tract obstruction [18]. In the present study, there was no tracheostomy and recurrence was managed surgically.

Three cases of bacterial tracheitis were diagnosed in 5-15 y children. All presented with fever, respiratory distress, cough, and stridor and required endotracheal intubation $(100 \%)$ with other supportive measures and antibiotics. One child grew Staphylococcus aureus in endotracheal secretion, while no organism was detected in the other 2 cases. In a recently published study by Casazza et al. out of 36 children with bacterial tracheitis, 69\% required PICU admission and 43\% required intubation [19].

Abnormalities in central nervous system can cause vocal cord paralysis. There was 1 case of Arnold-Chiari malformation, who presented at $8 \mathrm{mo}$ of age with complaints of noisy breathing since 4 mo and swelling over the lower back since birth. Stridor subsided after tracheostomy, and later surgery for underlying pathology, was carried out. Tracheostomy was decanulated after 2 mo. Yamada et al. in their study found stridor in all 12 children with Arnold-Chiari malformation. Stridor mostly started between $8 \mathrm{~d}$ to 3 mo of age but was most severe at 2-5 mo of age [20]. Bilateral VCP can cause respiratory distress presenting at birth, whereas unilateral VCP is usually asymptomatic. Etiology usually corresponds with birth trauma or neurological abnormality. Murty et al. estimated the incidence of bilateral VCP to be 0.75 cases per million births per year [21]. As spontaneous improvement is noted in almost $70 \%$ cases of bilateral VCP, one-year interval is given before surgery [22]. In the present study a 5-d-old neonate with bilateral VCP due to birth trauma was tracheostomized and he improved spontaneously within 6 mo. Another case of a 12-y-old boy with traumatic bilateral VCP due to road traffic accident required emergency tracheostomy and Kashima operation (Fig. 1d). Child was decanulated after 2 wk. In a retrospective study of $102 \mathrm{VCP}$ children, in $36(35.3 \%)$ patients, no specific cause could be identified. Among these, 26 had bilateral VCP and 10 had unilateral VCP. Twenty-eight patients $(78 \%)$ presented with symptoms from birth; in 7 (19\%), symptoms developed within the first 3 mo [22].

One patient of anaphylaxis presented with stridor where the allergen was suspected to be azithromycin. He improved with conservative management. Wheeze is the characteristic finding in anaphylaxis, but stridor can be found when upper airway is affected and though stridor is less common, it is much more dangerous. A recent study found stridor in only $6.3 \%$ of cases of anaphylaxis [23].

Subglottic stenosis may be congenital or acquired; most common acquired causes being postendotracheal intubation. A 12-y-old girl, who was tracheostomized 2 mo back for Guillain-Barre syndrome presented with stridor after $1 \mathrm{wk}$ of decanulation. Rigid laryngoscopic examination revealed grade III subglottic stenosis (Fig. 1e). She was retracheostomized and was referred to higher center for laryngotracheal reconstruction. Other case of subglottic stenosis was due to prolonged intubation and blunt trauma of trachea due to road traffic accidents. Both the children had grade II Myers-Cotton subglottic stenosis. Though the authors tried for endoscopic dilation and stenting with endotracheal tube, the stent got displaced. Finally, both cases were managed with permanent tracheostomy considering the cost factor. Kockar et al., in their study of 60 patients of extubation failure with inspiratory stridor, found subglottic stenosis in $12(20 \%)$ cases [24]. They did only tracheostomy in 6 patients and laser incision with topical mitomycin in 6 others. All were successfully decanulated and asymptomatic in the follow-up.

Laryngeal web is a congenital condition accounting for $2 \%-4 \%$ of laryngeal anomalies [25]. They usually present at birth or in first few months of life, but can also present in childhood. Benjamin in his series of 29 patients of laryngeal web, found only 1 child after infancy [26]. In the present study, web in a 5-y-old boy presenting with stridor and respiratory distress was found. He required emergency tracheostomy and later the thin web between two vocal cords was incised.

Tumors constituted very less number of cases with stridor $(1.49 \%)$. A 13-y-old boy who presented with left-side neck swelling since 2 mo and progressive noisy breathing was diagnosed to have anterior mediastinal mass compressing trachea from C7-D2 on imaging (Fig. 1f). As the child deteriorated, emergency intubation was done and he was ventilated mechanically. Further evaluation and treatment of the underlying pathology could not be carried out as the patient left against the medical advice. Mehrotra et al. had reported a child who had been treated as recurrent croup for $4 \mathrm{wk}$ and later diagnosed as mediastinal mass. They had proposed that a child presenting with 'recurrent croup' might warrant further investigation, so that underlying malignancy will not be missed [27].

Significant changes in etiology of stridor in children were noted when compared with the study conducted $30 \mathrm{y}$ back in CMC, Vellore [28]. Stridor due to diphtheria and acute epiglottis were significant during that time, while not a single case of stridor due to diphtheria or epiglottitis was found. This is most probably due to successful implementation of Universal Immunisation Program. However, the other causes of stridor were almost comparable. 


\section{Conclusion}

Pediatric stridor is an emergency, and sometimes lifethreatening and may warrant urgent interventions like intubation, bronchoscopy and tracheostomy. Pediatric stridor management is a teamwork between ENT surgeons, pediatric intensivist, pediatric surgeons, and anesthetists. A thorough knowledge regarding etiology, differential diagnosis, clinical and radiological evaluation, and airway management is required to deal with such cases in children. Securing airway is of utmost importance and precise management of cause can be carried out only after securing airway.

Author's Contribution SP, KKS conceived the design, analyzed and interpreted the data. MKJ, GZ, SP collected the data and helped in analysis of data. MKJ, GZ, SP drafted the article and SP, KKS revised it critically. All approved the final revised version of the article. KKS will act as guarantor for this paper.

\section{Declarations}

Conflict of Interest None.

\section{References}

1. Selvam DK, Vikram VJ, Saktheeswaran M, Mohammed IMP. A study on paediatric stridor causes and management: case series. Int J Otorhinolaryngol Head Neck Surg. 2017;3(4):1031-5.

2. Holinger L. Etiology of stridor in the neonate, infant and child. Ann Otol Rhinol Laryngol. 1980;89(5):397-400.

3. Chan PW, Goh A, Lum L. Severe upper airway obstruction in the tropics requiring intensive care. Pediatr Int. 2001;43:53-7.

4. Claes J, Boudewyns A, Deron P, et al. Management of stridor in neonates and infants. B-ENT. 2005;1:113-25.

5. Tunkel D, Zalzal G. Stridor in infants and children. Clin Pediatr. 1992;31(1):48-55.

6. Gelbart B, Parsons S, Sarpal A, Ninova P, Butt W. Intensive care management of children intubated for croup: a retrospective analysis. Anaesth Intensive Care. 2016;44(2):245-50. https://doi.org/10. 1177/0310057X1604400211.

7. Korlacki W, Korecka K, Dzielicki J, et al. Foreign body aspiration in children: diagnostic and therapeutic role of bronchoscopy. Pediatr Surg Int. 2011;27(8):833-7. https://doi.org/10.1007/ s00383-011-2874-8.

8. Shivakumar AM, Naik AS, Prashanth KB, Shetty KD, Praveen DS. Tracheobronchial foreign bodies. Indian J Pediatr. 2003;70(10): 793-7. https://doi.org/10.1007/BF02723797.

9. Yang X, Ni X, Guo Y, Zhang J. Combined low tracheotomy and rigid bronchoscopy to remove an irregular tracheal foreign body. Pediatr Investig. 2018;2(3):196-7.

10. Ramírez-Figueroa J, Gochicoa-Rangel L, Ramírez-San Juan D, Vargas M. Foreign body removal by flexible fiberoptic bronchoscopy in infants and children. Pediatr Pulmonol. 2005;40(5):392-7.
11. Vijayasekaran D, Gowrishankar N, Kalpana S, Vivekanandan V, Balakrishnan M, Suresh S. Lower airway anomalies in infants with laryngomalacia. Indian J Pediatr. 2010;77(4):403-6.

12. Fattah H, Gaafar A, Mandour Z. Laryngomalacia: diagnosis and management. Egypt J Ear, Nose, Throat Allied Sci. 2011;12(3): 149-53.

13. Denoyelle F, Mondain M, Gresillon N, et al. Failures and complications of supraglottoplasty in children. Arch Otolaryngol Head Neck Surg. 2003;129:1077-80.

14. Thakur S, Singh SK, Roy R, et al. Management of stridor in paediatric population-our experience. J Evol Med Dent Sci. 2019;8(48):3631-5.

15. Jayaram S, Marnane C. Pharyngitis. In: Watkinson JC, Clarke RW, editors. Scott-Brown's Otorhinolaryngology and Head and Neck Surgery. Boca Raton: CRC Press; 2019. p. 796.

16. Naik SM, Ravishankara S, Appaji M, Gautham MK, Devi P, Naik SS. Interval tonsillectomy: 27 cases of peritonsillar abscesses managed in medical college hospital. Otolaryngol Online J. 2013;3(4): 44-56.

17. James M, Katundu D, Chussi D, Shija P. Prevalence, clinical presentations, associated risk factors and recurrence of laryngeal papillomatosis among inpatients attended at a tertiary hospital in northern zone Tanzania. Pan Afr Med J. 2018;30:209.

18. Cole R, Myer C, Cotton R. Tracheotomy in children with recurrent respiratory papillomatosis. Head Neck. 1989;11(3):226-30.

19. Casazza G, Graham ME, Nelson D, Chaulk D, Sandweiss D, Meier J. Pediatric bacterial tracheitis - a variable entity: case series with literature review. Otolaryngol Head Neck Surg. 2019;160(3):5469.

20. Yamada H, Tanaka Y, Nakamura S. Laryngeal stridor associated with the Chiari II malformation. Childs Nerv Syst. 1985;1:312-8.

21. Murty GE, Shinkwin C, Gibbin KP. Bilateral vocal fold paralysis in infants: tracheostomy or not? J Laryngol Otol. 1994;108(4):329 31.

22. Daya H, Hosni A, Bejar-Solar I, Evans JN, Bailey CM. Pediatric vocal fold paralysis: a long-term retrospective study. Arch Otolaryngol Head Neck Surg. 2000;126(1):21-5.

23. Dribin TE, Michelson KA, Zhang Y, Schnadower D, Neuman MI. Are children with a history of asthma more likely to have severe anaphylactic reactions? A retrospective cohort study. J Pediatr. 2020;220:159-64.

24. Kockar T, Oktem S. A rare cause of stridor in children: acquired subglottic stenosis. Eur Respir J. 2017;50:PA4164. https://doi.org/ 10.1183/1393003.congress-2017.PA4164.

25. Fearon B, Ellis D. The management of long term airway problems in infants and children. Ann Otol Rhinol Laryngol. 1971;80(5): 669-77.

26. Benjamin B. Congenital laryngeal webs. Ann Otol Rhinol Laryngol. 1983;92(4):317-26.

27. Mehrotra S, Kilgar J, Lim R. Case 3: stridor and cough in a young child. Paediatr Child Health. 2014;19(10):523-5.

28. Rupa V, Raman R. Aetiological profile of paediatric laryngeal stridor in an Indian hospital. Ann Trop Pediatr. 1991;11(2):137-41.

Publisher's Note Springer Nature remains neutral with regard to jurisdictional claims in published maps and institutional affiliations. 